\title{
Avaliação de métodos para estimativa de coeficientes da cultura de amendoim
}

\author{
Luiz C. Silva ${ }^{1}$ \& Tantravahi V. R. Rao ${ }^{2}$
}

\begin{abstract}
RESUMO
A cultura do amendoim é uma alternativa agrícola importante para os perímetros irrigados do Nordeste brasileiro, em especial aqueles localizados em áreas de solos de textura arenosa, por sua baixa exigência hídrica, ciclo curto e uma demanda não atendida no mercado regional. Realizou-se este trabalho buscando-se avaliar diferentes métodos que determinam as necessidades hídricas da cultura do amendoim. O consumo hídrico da cultura foi determinado através de medições em evapotranspirômetros de lençol freático constante. A evapotranspiração de referencia foi obtida através de diferentes métodos. Constatou-se que a demanda hídrica da cultura é da ordem de $650 \mathrm{~mm}$ e o coeficiente de cultura (kc) obtido pelos métodos do Tanque Classe A, Hargreaves e Thornthwaite, são os mais adequados para o manejo eficiente da irrigação.
\end{abstract}

Palavras-chave: evapotranspiração, irrigação, Arachis hypogaea

\section{Evaluation of the methods of estimating the peanut crop coefficients}

\begin{abstract}
The peanut crop with its low water requirement, short growing period and a higher demand, which is not attended by the regional markets, is an important alternative crop to be grown in the irrigated areas of the Brazilian Northeast, especially in the sandy soils. This paper is concerned with the evaluation of different methods, which determine the water requirements of a peanut crop. The crop water consumption is determined from two evapotranspirometers with constant water table. The reference evapotranspiration is obtained by using different methods. The peanut crop water requirement is in the order of $650 \mathrm{~mm}$ and the crop coefficient obtained from the methods of Class A Pan evaporimeter, Hargreaves and Thornthwaite, is more adequate for efficient management of irrigation.
\end{abstract}

Key words: evapotranspiration, irrigation, Arachis hhypogaea 


\section{INTRODUÇÃO}

As áreas áridas e semi-áridas do Nordeste brasileiro caracterizam-se por acentuada variabilidade temporal e espacial das precipitações pluviais, contribuindo para o alto risco da agricultura de sequeiro e obtenção de baixas produtividades das culturas exploradas. A irrigação é uma prática que permite a redução dos riscos e possibilita que as culturas externem os seus potenciais de produtividade.

A cultura do amendoim (Arachis hypogaea L.) é uma importante alternativa para a agricultura irrigada da região nordestina, pela ampla demanda de consumo, condições edafoclimáticas adequadas e apresentar baixas necessidades hídricas para obtenção de boas colheitas que, segundo Doorenbos \& Kassam (1994), necessita de aplicação de 500 a $700 \mathrm{~mm}$ de chuva no período total de crescimento. Para Baldwin \& Harrison (1996) a cultura exige de 508 a $635 \mathrm{~mm}$ de água durante todo o ciclo, para expressar produtividade máxima. Nas condições do semi-árido do Nordeste brasileiro, Silva (1997) obteve as maiores produtividades, quando aplicou $700 \mathrm{~mm}$ de água durante o ciclo total da cultura.

No que se refere ao manejo de irrigação, a base para a quantificação da água a ser aplicada a dada cultura está associada, comumente, à capacidade da superfície, solo, e vegetação e atmosfera, de perder água em determinada condição climática. A forma usual de se quantificar a água a ser aplicada ao longo do ciclo da cultura, é considerar os processos de evaporação do solo e de transpiração da planta conjuntamente, no que se denomina evapotranspiração. Para a determinação da evapotranspiração, várias técnicas de medidas e modelos de estimativa têm sido propostas e usadas. As medidas são obtidas por meio de tanque evapotranspirométrico ou lisímetro de pesagem. Os modelos são expressões algébricas que tentam descrever os processos físicos relacionados com o fluxo de vapor d’água, em termos de variáveis climáticas e da vegetação.

A magnitude da evapotranspiração varia no tempo e no espaço, sendo a transpiração e a evaporação de alta sensibilidade às variações microclimáticas; esta é, portanto, uma das razões pela qual muitos métodos de estimativa não são particularmente precisos (Knapp, 1985). O estágio real de desenvolvimento da cultura deve ser considerado para que se obtenha maior eficiência na irrigação (Boote, 1982). Para Baldwin \& Harrison (1996) existem três períodos críticos às condições de disponibilidade de água, em que excesso ou escassez prejudica igualmente a produção do amendoim. O primeiro período compreende a fase de semeadura e germinação, o segundo a fase de formação das vagens e o terceiro a fase de maturação dos frutos. Assim, a estimativa e a obtenção de informações a respeito do consumo hídrico nas diversas fases do crescimento e do desenvolvimento da cultura do amendoim para as condições climáticas do semi-árido nordestino, racionalizam o uso da água, através de um planejamento de irrigação e reduz o desperdício de insumos, possibilitando maior rentabilidade da produção.

Foi objetivo do presente trabalho identificar o método de estimativa da evapotranspiração mais adequado às condições de cultivo do amendoim, e descrever as necessidades hídricas da cultura em cada estágio fenológico de seu desenvolvimento, por meio da determinação de coeficientes de cultivo (kc) fornecendo subsídios à agricultura irrigada.

\section{MATERIAL E MÉTODOS}

O experimento foi conduzido em condições de campo na Estação Experimental da Companhia de Desenvolvimento do Vale do São Francisco (CODEVASF) situada no município de Rodelas, BA, cujas coordenadas geográficas são $8^{\circ} 50^{\prime} \mathrm{S}$ de latitude; $38^{\circ} 46^{\prime} \mathrm{W}$ de longitude e altitude de $270 \mathrm{~m}$.

De acordo com a classificação de Köppen, o clima local é do tipo BSwh, correspondente a um clima muito quente, semi-árido e com estação chuvosa limitada aos meses de janeiro a abril, sendo que as precipitações são incertas e mal distribuídas.

O solo, classificado como Regossol (Neossolo Regolítico), de textura arenosa, cuja análise física realizada no Laboratório de Salinidade do Departamento de Engenharia Agrícola da Universidade Federal da Paraíba, UFPB, determinou $89,3 \%$ de areia, 3,2\% de silte e 7,5\% de argila e densidade aparente $1500 \mathrm{~kg} \mathrm{~m}^{-3}$.

A cultivar objeto do estudo foi a BR-1, de porte ereto, crescimento indeterminado, apta à colheita dos 90 aos 110 dias, indicada para agricultura de sequeiro. A semeadura foi realizada com espaçamento de $0,50 \mathrm{~m}$ entre fileiras e densidade de plantio de dez plantas por metro linear, como recomendam Silva et al. (1993). A cultura foi mantida livre das plantas daninhas, pragas e doenças durante todo o ciclo e conduzida sob regime de irrigação por aspersão. Para as irrigações utilizou-se um sistema fixo de aspersão, com distância de 12 m entre aspersores. A aplicação da água foi efetivada considerando-se uma eficiência do sistema aspersivo de $70 \%$, cujos aspersores aplicam em média, lâmina de $8 \mathrm{~mm} \mathrm{~h}^{-1}$.

O consumo hídrico da cultura (ETc) foi determinado através de dois evapotranspirômetros de lençol freático constante, com área de 2,25 m² cada, com a mesma configuração de plantio da área externa. A estimativa da evapotranspiração de referência (ETo) foi obtida através dos métodos do Tanque Classe A, Hargreaves (1977), Blaney \& Criddle (1950), Linacre (1977), Benavides \& Lopez (1970) e Thornthwaite (1954).

Para a obtenção das informações necessárias à estimativa da ETo, utilizaram-se o Tanque Classe A, instalado em uma Estação Meteorológica anexa à área experimental, psicrômetros de ventilação natural, fornecendo temperatura seca e úmida do ar, anemômetros instalados em uma torre no centro da área experimental, a $2 \mathrm{~m}$ de altura, medindo a velocidade do vento. O coeficiente de cultura $(\mathrm{kc})$ foi expresso pela relação: kc = ETc/ETo deduzida da expressão ETc = kc x ETo, para período semanal e para as fases fenológicas definidas pela FAO (Doorenbos \& Kassam, 1994).

Na análise dos dados utilizou-se, para a comparação dos métodos, o teste não paramétrico de Kruskal-Wallis, a 5\% de probabilidade (Campos, 1979). 


\section{RESULTADOS E DISCUSSÃO}

Os valores semanais das estimativas da evapotranspiração de referência (ETo) obtidos através dos diferentes métodos usuais para as regiões semi-áridas, da evapotranspiração máxima (ETc) medidos nos tanques de evapotranspiração e do coeficiente de cultivo (kc) determinados em função dos valores de ETo dos diversos métodos, encontram-se descritos na Tabela 1.

Constata-se que até a metade do ciclo da cultura, 6 a 7 semanas, os valores de ETo estimados pela maioria dos métodos, são superiores aos da ETc e que, após a sétima semana, o processo é inverso (Tabela 1).

O consumo de água nos evapotranspirômetros (ETc) foi menor durante o início do crescimento vegetativo, com média de $\cong 5 \mathrm{~mm} \mathrm{~d}^{-1}$, aumentando nas fases finais do crescimento da cultura, totalizando $648 \mathrm{~mm}$. O consumo máximo diário foi de cerca de $13 \mathrm{~mm}$ medido nas décima segunda e décima terceira semanas do ciclo da cultura. Esta alta taxa evapotranspiratória deveu-se, possivelmente, tanto pelo máximo índice de área foliar atingido pela cultura, quanto pela pronunciada demanda evaporativa da região, em associação aos efeitos de advecção de ar quente e seco na direção horizontal. Cândido (1998) trabalhando com amendoim na mesma área, também observou elevada taxa da evapotranspiração média diária, $11 \mathrm{~mm} \mathrm{dia}{ }^{-1}$, e associou o fenômeno às causas conjugadas da fase do crescimento da cultura e do acentuado efeito advectivo do ar seco. Os métodos menos precisos para se estimar as necessidades hídricas da cultura do amendoim, foram o de Benavides \& Lopez, Blaney \& Criddle e Linacre. Os demais estimaram, com razoável precisão, as necessidades de água do amendoim, dentro da amplitude sugerida por Doorenbos \& Kassam (1994) e são coerentes com a quantidade de água exigida para obtenção de maiores produtividades com a cultura na região em estudo (Silva \& Beltrão, 2000). O método mais simples e fácil de
Tabela 2. Comparação dos métodos de estimativa da ETo, com a ETc, pelo teste de Kruskal-Wallis

\begin{tabular}{cccc}
\hline Comparação & $\mathbf{P}$ & Comparação & $\mathbf{P}$ \\
(LIN e HAR) & $<0,01$ & (TCA e B\&L) & $<0,01$ \\
(LIN e TCA) & $<0,01$ & (TCA e THO) & $=0,10$ \\
(LIN e B\&L) & $<0,01$ & (TCA e B\&C) & $<0,01$ \\
(LIN e THO) & $<0,01$ & (TCA e ETC) & $=0,36$ \\
(LIN e B\&C) & $<0,01$ & (B\&L e THO) & $<0,01$ \\
(LIN e ETC) & $<0,01$ & (B\&L e B\&C) & $=0,25$ \\
(HAR e TCA) & $=0,50$ & (B\&L e ETC) & $<0,01$ \\
(HAR e B\&L) & $<0,01$ & (TH0 e B\&C) & $<0,01$ \\
(HAR e TH0) & $=0,35$ & (TH0 e ETC) & $=0,48$ \\
(HAR e B\&C) & $<0,01$ & (B\&C e ETC) & $<0,01$ \\
(HAR e ETc) & $=0,81$ & - & - \\
\hline
\end{tabular}

se aplicar, o do Tanque Classe A, foi o que mais se aproximou no total $(578 \mathrm{~mm})$ do valor medido, ETc $(648 \mathrm{~mm})$.

Para a tomada de decisão quanto ao método ou métodos a serem selecionados como os mais indicados para a estimativa da ETo utilizaram-se, para a comparação dos mesmos, o teste não paramétrico de Kruskal-Wallis, a 5\% de probabilidade, cujo resumo dos resultados se encontra na Tabela 2.

Conforme se observa na Tabela 2, os métodos que não diferem da ETc ao nível de $5 \%$ de probabilidade, são os de Hargreaves (HAR), Tanque Classe A (TCA) e Thornthwaite (THO) sendo, portanto, os métodos mais indicados para a estimativa da ETo na região do baixo São Francisco, nas margens da barragem de Itaparica. A decisão por um dos três métodos deverá ser tomada de acordo com a disponibilidade de recursos materiais, financeiros, humanos e tempo para a coleta das informações necessárias a cada método.

A variação estacional dos valores de kc obtidos pelos métodos de Linacre, Hargreaves, Tanque Classe A, Benavides \& Lopez, Thornthwaite, Blaney \& Criddle para as diferentes

Tabela 1. Evapotranspiração de referência (ETo) estimada pelos métodos de Linacre (LIN), Hargreaves (HAR), Tanque Classe A (TCA), Benavides \& Lopez (B\&L), Thornthwaite (THO), Blaney \& Criddle (B\&C) medida da evapotranspiração da cultura (ETc) e coeficientes de cultivo (kc) do amendoim para períodos semanais

\begin{tabular}{|c|c|c|c|c|c|c|c|c|c|c|c|c|c|}
\hline \multirow{2}{*}{ Semana } & \multicolumn{2}{|c|}{ LIN } & \multicolumn{2}{|c|}{ HAR } & \multicolumn{2}{|c|}{ TCA } & \multicolumn{2}{|c|}{ B\&L } & \multicolumn{2}{|c|}{ THO } & \multicolumn{2}{|c|}{$B \& C$} & \multirow{2}{*}{ ETc } \\
\hline & ETo & kc & ETo & kc & ETo & kc & Eto & kc & ETo & kc & ETo & kc & \\
\hline 1 & 5,11 & 0,96 & 5,79 & 0,85 & 5,87 & 0,84 & 6,26 & 0,78 & 4,77 & 1,03 & 4,21 & 1,17 & 4,94 \\
\hline 2 & 5,55 & 0,95 & 6,57 & 0,80 & 6,49 & 0,81 & 4,18 & 1,27 & 5,80 & 0,91 & 4,36 & 1,22 & 5,32 \\
\hline 3 & 5,56 & 0,93 & 6,83 & 0,77 & 7,27 & 0,72 & 4,20 & 1,25 & 5,70 & 0,92 & 4,35 & 1,20 & 5,26 \\
\hline 4 & 5,64 & 0,82 & 6,71 & 0,69 & 6,73 & 0,69 & 4,29 & 1,08 & 6,33 & 0,73 & 4,41 & 1,05 & 4,65 \\
\hline 6 & 5,49 & 1,02 & 6,10 & 0,91 & 7,20 & 0,77 & 4,48 & 1,25 & 8,48 & 0,66 & 4,63 & 1,21 & 5,61 \\
\hline 7 & 6,06 & 1,12 & 6,82 & 1,00 & 7,72 & 0,88 & 4,35 & 1,56 & 6,83 & 0,99 & 4,51 & 1,51 & 6,82 \\
\hline 8 & 5,72 & 1,40 & 6,59 & 1,22 & 5,72 & 1,40 & 4,30 & 1,87 & 6,72 & 1,19 & 4,50 & 1,78 & 8,05 \\
\hline 9 & 5,22 & 1,40 & 6,28 & 1,17 & 5,17 & 1,42 & 4,35 & 1,69 & 7,30 & 1,00 & 4,55 & 1,61 & 7,36 \\
\hline 10 & 4,45 & 1,81 & 5,34 & 1,51 & 5,50 & 1,47 & 3,69 & 2,19 & 4,33 & 1,86 & 4,29 & 1,88 & 8,09 \\
\hline 11 & 4,46 & 2,55 & 5,55 & 2,05 & 6,49 & 1,75 & 3,75 & 3,04 & 4,59 & 2,48 & 4,34 & 2,62 & 11,41 \\
\hline 13 & 5,12 & 2,53 & 6,45 & 2,01 & 6,37 & 2,04 & 4,02 & 3,23 & 5,34 & 2,43 & 4,42 & 2,94 & 13,00 \\
\hline Total & 479 & - & 561 & - & 579 & - & 386 & - & 557 & - & 391 & - & 648 \\
\hline
\end{tabular}


Tabela 3. Coeficientes de cultivo $(k c)$ obtidos pelos métodos de Linacre (kcLIN), Hargreaves (kcHAR), Tanque Classe A (kcTCA), Benavides \& Lopez $(k c B \& L)$, Thornthwaite $(k c T H O)$, Blaney \& Criddle $(k c B \& C)$ para as diferentes fases fenológicas da cultura (I - estabelecimento; II - período vegetativo; III - floração e formação das vagens; e IV - maturação)

\begin{tabular}{ccccccc}
\hline Fase & kcLIN & kcHAR & kcTCA & kcB\&L & kcTHO & kcB\&C \\
I & 0,96 & 0,85 & 0,84 & 0,79 & 1,03 & 1,17 \\
II & 0,90 & 0,76 & 0,74 & 1,20 & 0,85 & 1,16 \\
III & 1,39 & 1,20 & 1,14 & 1,77 & 1,13 & 1,65 \\
IV & $\mathbf{2 , 2 5}$ & $\mathbf{1 , 8 8}$ & $\mathbf{1 , 9 0}$ & $\mathbf{3 , 0 0}$ & $\mathbf{1 , 8 7}$ & $\mathbf{2 , 8 5}$ \\
\hline
\end{tabular}

fases fenológicas da cultura (I - estabelecimento; II - período vegetativo; III - floração e formação das vagens e IV - maturação), está descrita na Tabela 3.

Em geral, os déficits de água durante o período vegetativo (II) ocasionam atrasos do florescimento e aumentam o ciclo da cultura, retardando a colheita e reduzindo a produtividade A fase de floração e formação das vagens (III) é muito sensível à deficiência hídrica, acarretando queda de flores, inibição da polinização ou surgimento de flores estéreis. A fase inicial de formação das vagens é especialmente sensível ao déficit de umidade.

Esses valores de coeficientes de cultivo (Tabela 3) são elevados se comparados aos da literatura (Kassam et al., 1975) para os períodos I e IV; no entanto, uma análise dos parâmetros ambientais caracteriza a área em estudo como uma região com bastante influência do efeito da advecção horizontal de calor, favorecido pela vegetação arbustiva e esparsa, e pela grande demanda energética, que contribuem para elevar os valores da evapotranspiração da cultura (Cândido, 1998). Leitão (1989) observou, na mesma região, no perímetro irrigado de Mandacaru, Juazeiro, BA, uma advecção de $23 \%$, ao estudar o balanço de energia numa cultura de soja irrigada. Por outro lado, para as fases II e III, são as mais sensíveis ao déficit de umidade. Os valores de kc estabelecidos com base nos métodos do Tanque Classe A, Thornthwaite e Hargreaves, encontram-se na faixa de valores preconizados na literatura, variando de 0,80 a 1,10 (Kassam et al., 1975). Com esses fatos, os valores de kc tornam-se coerentes e podem ser aplicados na prática.

\section{CONCLUSÕES}

1. A necessidade hídrica total, $648 \mathrm{~mm}$, do amendoim cV. BR1, equipara-se à de outras cultivares.

2. No consumo de água, caracterizam-se três fases distintas, nas quais se evidencia que da emergência até o início da floração, o consumo é relativamente baixo; da floração à formação das vagens o consumo hídrico é crescente e proporcional ao crescimento e desenvolvimento da cultura; da formação das vagens até a maturação das sementes o consumo hídrico é estável e totalmente dependente das condições ambientais.

3. Os valores do consumo hídrico total da cultura estimado pelos métodos do Tanque Classe A, Hargreaves e Thornthwaite são coerentes com os medidos para a região em estudo que é da ordem de $650 \mathrm{~mm}$.
4. Os kc's mais representativos para o manejo eficiente de água da cultura são os obtidos em função da ETo estimada pelos métodos do Tanque Classe A, Hargreaves e Thornthwaite, sendo o do Tanque Classe A o mais indicado.

\section{LITERATURA CITADA}

Baldwin, J. A.; Harrison, K. A. Determining water use in peanut production. Irrigation Journal, Chicago, v.46, n.6, p.18-21, 1996.

Benavides, J. G.; Lopez, D. Formula para el caculo de la evapotranspiracion potencial adaptada al tropico $\left(15^{\circ} \mathrm{N}-15^{\circ} \mathrm{S}\right)$, Agronomia Tropical, Maracay, v. 20, n. 5, p.335-345, 1970.

Blainey, H. F.; Criddle, W. D. Determining water requirements in irrigated areas from climatological data.Technical Publication No. 96, Washington, DC. Soil Conservation Service, 1950.

Boote, K. J. Growth stages of peanut (Arachis hypogaea). Peanut Science, Stillwater. v.9, p.35-40, 1982.

Campos, H. de. Estatística experimental não paramétrica. $3^{\mathrm{a}}$ ed. Piracicaba: ESALQ/USP. 1979. 343p.

Cândido, L. A. Avaliação das necessidades hídricas e manejo de irrigação da cultura de amendoim. Campina Grande: UFPB, 1998, 75p. Dissertação Mestrado

Doorenbos, J.; Kassam, A. H. Efeito da água no rendimento das culturas Estudos de FAO: Irrigação e Drenagem, 33, Campina Grande: UFPB, 306p, 1994.

Hargreaves, G. H. World water for agriculture. Contract AID/taC-1103, Washington, DC: Utah State University and Agency for International Development, 177p, 1977.

Kassam, A. H.; Kowal, J. M.; Harkness, C. Water use and growth of groundnut at Samaru, Northern Nigeria. Tropical Agriculture. Guilford, v.52, n.2, p.105-111, 1975.

Knapp, H. V. Evaporation and Transpiration. In: Houghton, David D. Handbook of Applied Meteorology. New York: John Wiley \& Sons, P. 537-554, 1985.

Leitão, M. M. V. B. R. Balanço de radiação e energia numa cultura de soja irrigada. Campina Grande: UFPB, 1989.110p. Dissertação Mestrado

Linacre, E. T. A simple formula for estimating evaporation rates in various climates, using temperature data alone. Agricultural Meteorology, Amsterdam, v.18, p.409-424, 1977.

Silva, L. C. Respostas ecofisiológicas e desempenho agronômico do amendoim cv. BR1 submetido a diferentes lâminas e intervalos de irrigação. Campina Grande: UFPB, 1997, 126p. Tese Doutorado

Silva, L. C.; Beltrão, N. E. de M. Incremento de fitomassa e produtividade do amendoinzeiro em função de lâmina e intervalos de irrigação. Revista de Oleaginosas e Fibrosas, Campina Grande, v.4, n.2, p.111-121, 2000.

Silva, L. C.; Moreira, J. de A. N.; Sobrinho, J. T.; Beltrão, N. E. de $\mathrm{M}$. Recomendações técnicas para o cultivo do amendoim no Nordeste brasileiro. Campina Grande: EMBRAPA - CNPA. p.13, 1993. Circular Técnica, n.16

Thoronthwait, C. W., 1954. A re-examination of the concept and measurement of potential evapotranspiration. In John R. Mather (ed.), "The measurement of potential evapotranspiration”, Publications in Climatology, v.7, p.200-209, 1954. Centerton, New Jersey. 